REVISTA ECONOMÍA

Vol. 69, N. ${ }^{\circ}$ IO9 (mayo), I89-2I2

\title{
ELEMENTOS DE ANÁLISIS PARA LA SOSTENIBILIDAD DE UNA ECONOMÍA DOLARIZADA
}

\author{
JAIME FERNÁNDEZ \\ Flacso, Ecuador
}

\begin{abstract}
Recepción manuscrito: 6 de enero de 2017
\end{abstract}
Aceptación versión final: 15 de abril de 2017

\begin{abstract}
RESUMEN La dolarización en Ecuador ha sido objeto de innumerables debates desde que se adoptó esta medida a inicios del año 2000. Las particularidades que presenta la economía ecuatoriana hacen que la evaluación de la sostenibilidad de la dolarización sea aún un campo abierto de investigación. En este artículo se presentan algunos elementos de análisis, principalmente desde un enfoque teórico, que deben ser tomados en cuenta para entender el funcionamiento de la dolarización, así como sus potenciales vulnerabilidades. En el documento se presentan también los resultados de dos escenarios de política, simulados a partir de un modelo stock-flow consistent teórico para una economía dolarizada.
\end{abstract}

PALABRAS CLAVE Dolarización, dinero endógeno, modelos stock-flow consistent.

ABSTRACT Dollarization in Ecuador has been the subject of innumerable debates since the adoption of this measure at the beginning of 2000. The peculiarities presented by the Ecuadorian economy make the evaluation of the sustainability of dollarization still an open field of research. This article presents some elements of analysis, mainly from a theoretical approach that must be taken into account to understand the operation of dollarization, as well as its potential vulnerabilities. The paper also presents the results of two policy scenarios, simulated from a theoretical stock-flow consistent model for a dollarized economy.

KEYWORDS Dollarization, endogenous money, stock-flow consistent models.

JEL CODES E42, E47, E51.

\section{INTRODUCCIÓN}

La profunda crisis que experimentó el Ecuador a finales de los años noventa tuvo su culmen con la dolarización de su economía. Esta medida, probablemente apresurada y poco planificada, se dio los primeros días de enero del 2000. Casi de inmediato, aparecieron fuertes voces que alertaban sobre las graves consecuencias que esta medida acarrearía en varias dimensiones del quehacer nacional: sociales, económicas, monetarias e incluso políticas. Más aún, desde el inicio de la dolarización se debatía en varias instancias académicas y oficiales la sostenibilidad del nuevo esquema monetario. Desde luego, como recuerda Acosta (2012), también hubo quienes afirmaban que el país ya tenía «un pie en el primer mundo» por haber adoptado la moneda norteamericana. 
Ha transcurrido más de una década y media desde que se adoptó la dolarización en Ecuador. Las discusiones han sido interminables alrededor de los beneficios y perjuicios que esta medida ha traído para el país. Por un lado, hay pensadores que defienden la medida e incluso le atribuyen buena parte del desarrollo que ha experimentado el país en este período. En contraparte, hay quienes ven en ella «más una camisa de fuerza que una salvación», como mencionan Sachs y Larrain (2000). Sin necesidad de profundizar en rigurosas demostraciones, se podría concluir que, salvo determinados períodos, el país experimentó una relativa estabilidad económica durante los años posteriores a la dolarización. Sin embargo, han sido esos períodos poco favorables los que, aún siendo relativamente cortos, han generado grandes dudas respecto de la sostenibilidad de la dolarización.

La dolarización, según menciona Falconí (2004), tiene dos peligros: la vulnerabilidad ante los flujos externos financieros y comerciales, y el desequilibrio de las finanzas públicas. En esta visión, apegada a la perspectiva de dinero exógeno, la sostenibilidad de la dolarización estaría condicionada únicamente por el saldo neto de ingresos y salidas de dólares de la economía. Esta disponibilidad de divisas en una economía dolarizada dependería de: la inversión extranjera, las remesas, los saldos de las balanzas de bienes, renta y servicios, la reserva internacional y la capacidad de recibir financiamiento externo. No obstante, desde una perspectiva de dinero endógeno, la identificación de factores que atenten contra la estabilidad de este esquema monetario requiere de un análisis mucho más profundo; en este artículo precisamente planteará la discusión desde esta visión.

En Ecuador, prácticamente todos los indicadores macroeconómicos relacionados a las variables citadas anteriormente, se han ido deteriorando de forma sistemática a partir de la crisis financiera mundial de 2008 2009. Más aún, a partir del nuevo descalabro de los precios del petróleo que inició a mediados de 2014, la situación se ha agudizado. Todos estos antecedentes conducen a cuestionar la sostenibilidad del esquema monetario actual. Es fundamental, entonces, entender a cabalidad el funcionamiento de una economía dolarizada como la ecuatoriana y cuáles serían los límites que podría tener su sostenibilidad.

\section{DOLARIZACIÓN EN SENTIDO AMPLIO}

Pese a que han transcurrido 17 años desde que el país adoptó la dolarización, los estudios rigurosos acerca de estos sistemas monetarios no son abundantes en la literatura especializada. De aquí, además de otras explicaciones que se mencionarán más adelante, surge la justificación para definir a la dolarización en un sentido más amplio. En un sentido tradicional, la dolarización, conocida como oficial, puede implementarse bajo dos modalidades: fijación del tipo de cambio a una moneda fuerte o sustitución de toda la divisa nacional por una extranjera, como el caso ecuatoriano. Sin embargo, la dolarización puede también ser entendida como la tenencia y utilización de activos y pasivos financieros en una moneda extranjera - no necesariamente el dólar-. Entendida la medida en este sentido amplio, existe una cantidad considerablemente mayor de investigaciones que aportan su entendimiento.

Sea cual fuere la forma de dolarización que se analice, parece haber un relativo consenso respecto de las motivaciones - mas no de los beneficios y desventajas que podrían existir detrás de 
un proceso de dolarización-. La principal motivación para adoptar un esquema monetario de este tipo, tanto de actores oficiales como privados, es la disminución de costos y riesgos asociados a una moneda local altamente volátil. Esta es la conclusión a la que han llegado varios autores que analizaron las condiciones monetarias y financieras de Latinoamérica y el Caribe durante la segunda mitad del siglo xx. La dolarización en esta región experimentó un primer boom hacia finales de los años setenta (Caprio, Honohan y Vittas, 2002). En varios países de la región, particularmente latinoamericanos, existía una creciente inflación durante la época. Era frecuente también encontrar un ritmo creciente de tenencia de activos en moneda extranjera, primero en el exterior y luego bajo la figura de depósitos en bancos locales. Incluso en países como Chile, Brasil y Colombia, en los que no se permitían depósitos en moneda extranjera, ésta era utilizada como medio de cambio en mercados paralelos y como medio para guardar valor.

Este proceso se aceleró en la región durante los años noventa. La caja de conversión en Argentina representa un caso paradigmático: este país sudamericano ancló su tipo de cambio al dólar estadounidense. Casos similares fueron los de Lituania y Estonia, aunque este último optó por fijar la paridad de su tipo de cambio al marco alemán. Estos procesos de dolarización, informal - privada - u oficial, se profundizaron en la región a inicios de los años 2000 con la adopción del dólar estadounidense como moneda oficial por parte de Ecuador y de El Salvador. No obstante, ninguno de los países mencionados alcanzaría la trascendencia y magnitud del más grande caso de dolarización que jamás haya tenido lugar: la adopción del euro por parte de la Unión Europea.

A primera vista, existirían grandes diferencias entre los primeros casos mencionados sobre todo los latinoamericanos y el de la Unión Europea-; sin embargo, un análisis más profundo de estas experiencias muestra que las semejanzas son quizás mayores que las diferencias. En principio, la adopción del euro responde a un proceso de larga data; de hecho, como señala Bell (2003), una unión monetaria y económica ya fue acordada en 1969 por los jefes de gobierno de los seis países fundadores de la Comunidad Económica Europea. Además, gracias a la creación de instituciones comunes, como el Banco Central Europeo, se esperaba que todos los países que adoptaron el euro tuvieran algún nivel de decisión en la política monetaria común.

De haberse concretado los objetivos originales de la integración europea, se habría marcado una notable diferencia con la situación de países como Ecuador o El Salvador, que no tienen injerencia alguna en las decisiones de política monetaria de la Reserva Federal de los Estados Unidos. No obstante, el paso del tiempo y las recientes crisis de algunos países de la periferia europea, como Grecia y Portugal por ejemplo, ha mostrado que estos países enfrentan amenazas similares a las de los países dolarizados de Latinoamérica y el Caribe: vulnerabilidad ante choques externos, falta de autonomía en la política monetaria, financiamiento condicionado, entre otros.

\section{HISTORIA DE LA DISCUSIÓN}

Para entender un proceso de dolarización, sus causas y efectos, y una potencial reversibilidad de este esquema monetario, es fundamental entender la relación entre producción, precios y dinero. Aún cuando se puede identificar una amplia variedad de escuelas del pensamiento que 
se aproximan de distinta manera a la relación entre el dinero y el sector «real» de la economía, se pueden identificar seis autores/momentos que han sido determinantes en esta discusión. De los fundamentos de las teorías formuladas por estos pensadores se han derivado prácticamente todas las construcciones teóricas que buscan explicar esta interacción.

\section{SAY Y LOS CLÁSICOS}

La cita que se reproduce a continuación, tomada de A treatise on political economy: or the production, distribution, and consumption of wealth (Say, 1836), refleja de forma clara y concisa el pensamiento de los primeros clásicos discípulos de Jean Baptiste Say:

Un producto terminado ofrece, desde ese preciso instante, un mercado a otros productos por todo el monto de su valor. En efecto, cuando un productor termina un producto, su mayor deseo es venderlo, para que el valor de dicho producto no permanezca improductivo en sus manos. Pero no está menos apresurado por deshacerse del dinero que le provee su venta, para que el valor del dinero tampoco quede improductivo. Ahora bien, no podemos deshacernos del dinero más que motivados por el deseo de comprar un producto cualquiera. Vemos entonces que el simple hecho de la formación de un producto abre, desde ese preciso instante, un mercado a otros productos. (s. p.)

Dicho de otro modo, en el pensamiento clásico posterior a Say, el dinero m cumplía únicamente la función de medio de cambio, que permitía transformar un bien $C$ en otro $C^{\prime}$ : C-M-C' (1)

\section{ESCUELA MONETARIA FRENTE A ESCUELA BANCARIA}

La British Banking School y la British Currency School fueron dos escuelas, dentro del seno del pensamiento económico clásico, que sostuvieron intensos debates en torno a aspectos que, a la postre, resultarían fundamentales para entender el dinero - algunos de los cuales continúan siendo puntos álgidos de discusión hasta la actualidad-. A continuación se muestra una breve síntesis de los principales postulados que sostenían ambas escuelas (Humphrey, 1974).

Por un lado, la Escuela Monetaria: i) solo las monedas y los billetes son dinero, ii) el stock de dinero determina la demanda agregada, y iii) la demanda agregada determina los precios. Por otro lado, la Escuela Bancaria: i) el dinero es visto como un medio de intercambio que es creado espontáneamente - de forma endógena en el mercado- entre los comerciantes, ii) la demanda agregada determina el stock de dinero, iii) si se necesita influenciar los precios en la economía, hay que controlar el crédito.

MARX

La ruptura de varias décadas de hegemonía del pensamiento clásico respecto de la función del dinero llegaría de la mano de Marx, además de una primera formulación de la teoría de demanda efectiva (Marx, 1893). Marx expresó la característica esencial del proceso de producción capitalista y de circulación con la relación: M-C-M’ (2).

Con esta formulación, Marx sostenía que el dinero no era simplemente un medio de intercambio para tranzar entre mercancías, sino que en sí mismo era un objetivo de acumulación fruto del proceso de producción capitalista. La lectura de esta formulación consiste en que al inicio del proceso de producción el capitalista introduce una cantidad inicial de dinero 
$M$, que hace posible dicho proceso. Con $M$ se adquiere trabajo e insumos, los mismos que se convierten en un bien $C$, que a su vez es vendido en el mercado para obtener una cantidad $M^{\prime}$ que, desde luego, ha de esperarse mayor a $M$.

Claramente, la existencia de un $M$ ' que sea sistemáticamente mayor a $M$ en cada repetición del circuito productivo demandaba una explicación mucho más profunda, acerca de la creación del dinero, que aquellas existentes hasta la época. Marx propuso, por tanto, tres posibles explicaciones: i) aumento en la velocidad de circulación del dinero, ii) disminución en la preferencia por la liquidez — transformación de dinero acumulado en dinero circulante, y ii) incremento en la oferta de dinero.

Las dos primeras explicaciones fueron descartadas por el propio Marx. En el primer caso, la velocidad de circulación del dinero no podía aumentar de forma indefinida para sostener el creciente ritmo de acumulación propio del sistema capitalista. Por otro lado, los dueños del capital podrían reducir su preferencia por la liquidez unas cuantas veces, de manera que su dinero acumulado se convierta en circulante - lo que era bastante factible en las economías del primer mundo gracias a los recursos que llegaban de las colonias-, pero éste tampoco podía ser un mecanismo sostenible de forma indefinida en el tiempo. Finalmente, Marx concluye que la única explicación viable es que la oferta de dinero se incremente - entre líneas se entiende que debe ser de forma endógena - en el tiempo al mismo ritmo al que crecía la producción. De hecho, esta última explicación da lugar a una primera teoría estructurada de la existencia de crisis por falta de demanda efectiva.

\section{LA TEORÍA CUANTITATIVA}

Desde la formulación original de la teoría cuantitativa del dinero, que se atribuye al filósofo del siglo XVI Jean Bodin (Humphrey, 1974), existieron importantes debates en torno a ésta, principalmente dentro del pensamiento económico clásico. Sobresalieron tres escuelas que debatían en torno a la teoría cuantitativa del dinero: la Bancaria y Monetaria previamente citadas, y la Escuela Bullionista. No obstante, la primera formulación formal de la teoría se remonta a Irving Fisher, de la escuela neoclásica. Fisher propuso una formulación matemática - llamada ecuación de cambio- que relacionaba cuatro magnitudes fundamentales de la economía (ver ecuación 3): oferta monetaria $(M)$, velocidad de circulación del dinero $(v)$, nivel general de precios $(P)$ y volumen físico de transacciones de una economía (

Alternativamente, la ecuación de cambio puede ser escrita según la formulación de Marshall y Pigou —ecuación de Cambridge-como: $\mathrm{M}=k \mathrm{P} y(4)$, donde $k$ es el ratio de efectivo deseado en la economía y es el ingreso nacional en términos reales. En ambas ecuaciones, se suponen $T$ y $V$, $o$ $y$ y $k$ constantes, según sea el caso, de manera que dados los precios y bajos supuestos tales como el hecho de que la economía estaba en pleno empleo, $M$ quedaba determinada. Precisamente, esta ecuación fue utilizada por la corriente neoclásica para teorizar acerca de la determinación de los precios de una economía y su relación proporcional con la oferta monetaria total.

\section{KEYNES}

Posteriormente, Keynes en los años treinta argumentaría en contra de esta teorización previa y rompería con décadas de una hegemonía casi irrefutable de dicho pensamiento. Keynes 
argumentaba, entre otras cosas, que solamente si la economía estaba produciendo a su capacidad total y con pleno empleo, entonces los cambios en la oferta monetaria alterarían únicamente al nivel de precios. Por otro lado, si la economía operaba con desempleo y por debajo de la capacidad total, entonces los cambios en la oferta monetaria afectarían al output y al nivel de empleo más que a los precios.

Keynes también argumentó que los precios eran determinados en buena parte por los costos asociados a los salarios, y que estos a su vez estaban determinados por aspectos institucionales, por lo que no se podían ajustar automáticamente. De este modo, Keynes reversó la lectura de la ecuación de cambio de Fisher. Además, un cambio en el volumen físico de las transacciones podría ser perfectamente absorbido por la velocidad de dichas transacciones, dejando así sin alteración la oferta monetaria. En resumen, Keynes argumentó fuertemente en contra de los rígidos vínculos entre dinero, velocidad de circulación, precios e ingreso nominal que proponía la teoría anterior.

\section{FRIEDMAN}

La tercera escuela de referencia dentro de la teoría cuantitativa del dinero llegaría años después de la mano de Friedman, quien reformuló dicha teoría y la convirtió en una forma de determinación de la demanda de dinero, en lugar de ser un mecanismo de determinación de los precios o del ingreso nominal. Al ser reinterpretada la teoría cuantitativa del dinero, ya no como una forma de determinación del ingreso nominal, Friedman pudo contrarrestar del algún modo la crítica de Keynes acerca del supuesto neoclásico del pleno empleo. Otra modificación implementada por Friedman, para «liberarse» de las críticas keynesianas consistió en la teorización acerca de la velocidad de circulación del dinero; el autor propuso que dicha velocidad estaba determinada por una relación estable con un pequeño número de variables independientes que no estaban explicitadas en la ecuación de cambio.

Como se mencionó antes, los tres autores revisados constituyen los pilares de un sinnúmero de debates que vendrían en décadas posteriores, entre los que se destacan extensas discusiones acerca de la neutralidad o no del dinero. Precisamente, esta discusión sería uno de los puntos fundamentales de divergencia entre distintas escuelas del pensamiento, tanto ortodoxas - síntesis neoclásica, ciclos económicos reales, nueva síntesis neoclásica- como heterodoxas — circuitistas, neocartalistas, estructuralistas-. Aunque se podría profundizar en cada una de estas escuelas sobre sus postulados, su forma de entender el dinero, la interacción entre los sectores monetario-financiero y real, entre otros aspectos, para efectos de esta investigación resulta más provechoso ahondar sobre un aspecto específico derivado de estas discusiones: teorías del tipo de cambio y, de forma más específica, adopción de un tipo de cambio fijo.

\section{TIPO DE CAMBIO FIJO FRENTE A FLEXIBLE}

Cualquier discusión acerca de la dolarización, en cualquiera de sus formas — privada, oficial con sustitución monetaria u oficial con caja de conversión-, necesariamente debe remitirse a un extenso debate acerca del tipo de cambio por el que debería optar una economía: fijo o flexible. En esta discusión necesariamente hay que remitirse a los trabajos de Mundell y Fleming en los años sesenta y Dornbusch en los años setenta. 
Mundell (1961) sostiene que un tipo de cambio flexible "protege» más a la producción interna ante shocks de demanda externa, mientras un tipo de cambio fijo lo hace ante shocks de demanda interna. Aunque no lo dice explícitamente Mundell, en este último caso si bien la producción interna puede estar más protegida ante shocks de demanda interna, también es considerablemente más vulnerable ante shocks externos. Una de las principales implicaciones del modelo de Mundell-Fleming-Dornbusch (MFD) es que, en presencia de tipos de cambio flexibles, cambios permanentes no anticipados en la demanda de dinero pueden impactar el sector real de la economía debido a la rigidez de los precios (Levy y Sturzenegger, 2003). Así, un incremento en la demanda de dinero - que no sea correspondido automáticamente por un incremento en la oferta-generaría una contracción temporal - mientras los precios se ajustan a la nueva demanda de saldos reales - con tasas de interés más altas y mayor desempleo.

Por otro lado, el modelo MFD plantea que un tipo de cambio fijo previene a la economía de efectos reales. Sin embargo, en este caso, un incremento de la oferta monetaria, se vería reflejado directamente en una variación de las reservas internacionales, dejando así sin efecto dicho shock. Consecuentemente, bajo este régimen de tipo de cambio, la política monetaria contracíclica estaría fuertemente limitada. En caso de existir shocks reales, bajo un régimen de tipo de cambio fijo requeriría un ajuste en los precios nominales locales. Si se considera la rigidez de los precios domésticos, existiría entonces un período de contracción de la demanda interna y un incremento en los niveles de desempleo, mientras dichos precios se ajustan.

Una suerte de síntesis de los trabajos de Mundell se encuentra en el famoso trilema de la economía internacional — también conocido como la trinidad imposible-. Según esta propuesta, cualquier economía debe escoger entre contar con dos, y solamente dos, de las siguientes características: i) tipo de cambio fijo, ii) cuenta de capital libre, y iii) política monetaria autónoma.

Estas discusiones acerca del tipo de cambio no solamente marcaron un camino en la política monetaria de varias regiones, sino que dieron lugar incluso a una posterior polarización de las posturas. Por un lado, varios países, impulsados también por tendencia a liberalizar el movimiento de capitales, se alinearon hacia un tipo de cambio completamente flexible: flotante. Otros países, en cambio, optaron por un tipo de cambio fijo anclado a monedas fuertes, o por uniones monetarias. Profundizando una vez más en los objetivos específicos de esta investigación, en la siguiente sección se revisarán tres posibles explicaciones mainstream a la dolarización, que se han desarrollado con base en los pilares teóricos previamente explicados: áreas monetarias óptimas, crisis monetarias por inestabilidad cambiaria y acentuada dolarización de facto.

\section{POSIBLES EXPLICACIONES ORTODOXAS A UN PROCESO DE DOLARIZACIÓN}

\section{ÁREAS MONETARIAS ÓPTIMAS}

La teoría de las áreas monetarias óptimas de Mundell (1961) es, quizás, la explicación canónica que desde la ortodoxia se pretende dar a un proceso de dolarización, nuevamente entendida 
en un sentido amplio como se dijo antes. Esta teoría sostiene que el tipo de cambio nominal puede ser utilizado como un instrumento para "proteger» a una economía frente a shocks reales; para lo cual la solución sería la integración monetaria. Los beneficios de contar con una unión monetaria consistirían en la disminución de costos transaccionales entre los países miembros de dicha unión; además, la magnitud disminución de costos estaría en relación directa con el nivel de comercio intraunión, es decir, a mayor comercio entre los países miembros mayor será el beneficio debido a la disminución de costos transaccionales. Desde luego, existe un costo a asumir como contraparte de este beneficio en los países miembros. Ante shocks reales, los países miembros ya no contarían con el tipo de cambio como medida de estabilización. No obstante, los defensores de esta visión argumentarían en favor de esta teoría aduciendo que estas desventajas se podrían reducir sistemáticamente: i) en la medida en que exista mayor movilidad de los factores entre los países de la unión (Mundell, 1961), y ii) mientras más «simétricos» sean los choques entre los países de la unión (Kenen, 1969).

Han existido varios intentos de aplicar esta teoría a los procesos de dolarización, oficial o no, que han experimentado los países de América Latina (Jameson, 2004). Sin embargo, en ninguno de ellos se ha podido justificar el cumplimiento de los requerimientos de base para que exista un área monetaria óptima, que en este caso debería incluir a Estados Unidos. Aunque existía un considerable nivel de comercio entre los países latinoamericanos y Estados Unidos, claramente no existía - ni existe - una libre movilidad de los factores entre esta región y Estados Unidos, al menos no en sentido bidireccional. Por otro lado, muy difícilmente, salvo en el caso de El Salvador, existía una estrecha relación entre los ciclos económicos de los países en proceso de dolarización y los de Estados Unidos. En conclusión, estos procesos de dolarización, y particularmente el ecuatoriano, muy difícilmente pueden ser explicados por la teoría de las áreas monetarias óptimas.

\section{CRISIS MONETARIAS POR INESTABILIDAD CAMBIARIA}

Las teorías de crisis monetarias sostienen que las economías pequeñas muy difícilmente pueden tener control sobre el tipo de cambio - estabilidad cambiaria - en períodos en los que se acentúa la libre movilidad de capitales. Dicho de otro modo, los altos niveles de volatilidad del tipo de cambio en un régimen de tipo de cambio flexible conducirían a que los países adopten una de dos posturas - la polarización a la que se hizo referencia anteriormente-: adoptar un tipo de cambio fijo o absolutamente flexible (flotante).

Una vez más, esta teoría no podría ser exitosamente contrastada empíricamente. A finales de los años noventa, el tipo de cambio era mucho más volátil en países que tenían menores niveles de dolarización no oficial que en aquellos que sí los tenían. Países como Brasil, Venezuela y Surinam tenían tipos de cambio sumamente volátiles (Frieden, 2003) y, sin embargo, no adoptaron un tipo de cambio fijo o avanzaron hacia una dolarización oficial. En contraparte, países como Perú o Bolivia, continuaron con una profundización de sus esquemas de dolarización de facto aun cuando su tipo de cambio era considerablemente menos volátil que el de los países mencionados. El caso ecuatoriano estaría aparentemente próximo a poder ser explicado por esta teoría, pues a finales de los años noventa efectivamente los elevados niveles de depreciación de la moneda local, junto con una galopante inflación, condujeron a la adopción del dólar 
estadounidense como moneda oficial. No obstante, como se verá más adelante, un análisis institucionalista histórico sería quizás más adecuado aún para explicar este proceso en Ecuador.

\section{ACENTUADA DOLARIZACIÓN DE FACTO}

Una tercera teorización ortodoxa que pretende explicar la dolarización está dada por los altos niveles de tenencia y circulación de divisa extranjera en una economía dolarización privada o no oficial. Según esta teoría, la dolarización oficial es el último estadio de una transición que empieza con un tipo de cambio flexible y pasa por una etapa de caja de conversión con tipo de cambio fijo, para finalmente desembocar en la sustitución total de la moneda local. Este tipo de dolarización de facto - también conocida como sustitución de moneda - tiene lugar cuando los hogares y firmas deciden optar por la tenencia de moneda extranjera para evitar la disminución del valor de sus tenencias en moneda local, principalmente debido a altos niveles de inflación o a bajos retornos reales a sus tenencias por parte de los bancos locales. Según se señala en Caprio, Honohan y Vittas (2002), esta teoría sostiene que los depósitos en moneda extranjera aumentan cuando la inflación es alta y el tipo de cambio se deprecia.

Estas teorías fueron ampliamente contrastadas empíricamente en Latinoamérica y el Caribe sin que necesariamente haya existido evidencia fuerte a su favor. Tal es el caso, por ejemplo, de países como Argentina, Perú, Bolivia o Uruguay, que llegaron a tener más del 50\% de sus depósitos en moneda extranjera - mayoritariamente dólares- a finales de los años noventa $y$, sin embargo, solo uno de ellos terminó adoptando un tipo de cambio fijo; medida que años más tarde sería revertida por los desastrosos efectos que tuvo en la economía de Argentina. Ecuador, aunque tenía también un porcentaje considerable de depósitos en moneda extranjera, estaba por debajo de los niveles de los países mencionados anteriormente, por lo que tampoco parece plausible esta explicación para su caso.

\section{POSIBLES EXPLICACIONES OUT OF THE STREAM}

Ante la falta de una explicación cabal de los procesos de dolarización en América Latina y, puntualmente, en Ecuador, en esta sección se revisan otras probables explicaciones que surgen de corrientes alternativas del pensamiento económico. En primer lugar, se presenta un análisis de economía política que cuenta con una validación empírica mediante técnicas econométricas. En segundo punto, se presenta un análisis poskeynesiano de corte institucionalista que, utilizando varias categorías de análisis, intenta explicar el proceso de dolarización en el caso ecuatoriano.

\section{ANÁLISIS DE ECONOMÍA POLÍTICA}

Frieden (2003) propone una potencial explicación a los procesos de dolarización en América Latina y el Caribe a partir de dos aristas: la distribución del poder y la situación política de los países. En cuanto al primer punto, el autor plantea que una dolarización será más viable, como es lógico, en la medida en que los grupos de poder resulten beneficiados por tal medida. En vista de que la dolarización aumentaría eventualmente el nivel de comercio internacional e inversiones en un país, las firmas que prevean ser beneficiadas de esta mayor integración apoyarán 
el nuevo régimen monetario. Rose (2000) estimó que las relaciones comerciales casi se triplicarían entre dos países que compartan una moneda común. Dicho de otro modo, las firmas con mayor propensión a incursionar o expandirse hacia los mercados internacionales estarán a favor de una eventual dolarización, mientras que las firmas que tendrían que competir localmente con importaciones probablemente no lo estarán; la adopción o no de una moneda extranjera dependerá entonces en buena medida de la distribución del poder entre unos y otros.

Por otro lado, los incentivos a los cuales estén sujetos los actores políticos de un país serán necesariamente determinantes de la decisión de adoptar o no una eventual dolarización. Así, por ejemplo, buena parte de la motivación para dolarizar un país se encontrará en la fortaleza o debilidad electoral del gobierno, así como en su habilidad para implementar una política de fijación de tipo de cambio o sustitución integral por una moneda extranjera; un gobierno con fuerte apoyo probablemente estará en mayor capacidad de implementar las medidas necesarias para sostener el nuevo régimen monetario. Desde luego, otros aspectos como la corrupción y la inestabilidad política juegan un papel preponderante en cuanto a la decisión de dolarizar o no una economía.

Aun cuando varias dimensiones de un análisis de economía política no son del todo susceptibles de ser incorporadas a una validación econométrica, Frieden (2003) ensaya un estudio empírico en el que busca validar algunas de sus hipótesis. El autor ajusta una regresión logística con datos de países latinoamericanos entre 1960 y 1994 para modelar la probabilidad de adoptar un tipo de cambio fijo, utilizando como variables explicativas: inflación, hiperinflación, nivel de apertura comercial, tamaño del sector manufacturero, inestabilidad política, fuerza del gobierno y fragmentación de la oposición. Los tres principales resultados de su estudio son: i) el haber experimentado hiperinflación en los últimos años aumenta en 21\% la probabilidad de dolarizar una economía, ii) un incremento de una desviación estándar en el nivel de apertura comercial aumenta la probabilidad en $25 \%$, y iii) el haber experimentado situaciones de alta inestabilidad política - al menos tres cambios de gobierno en los últimos cinco años o al menos dos en los últimos tres años- incrementa la probabilidad en 19\%.

Con base en la estimación econométrica anterior, el autor estima las probabilidades de adoptar la dolarización para 26 países de América Latina y el Caribe. Si se ordenan en orden descendente dichas probabilidades, Argentina está en el penúltimo puesto, es decir, era el segundo país con menor probabilidad de dolarizarse, El Salvador está en el puesto 20 y Ecuador en el puesto 18. Es decir, hay aproximadamente 20 países que están por encima de estos tres países, que serían los que a la postre terminarían adoptando la medida de dolarizar sus economías. Desde luego, estos resultados no invalidan necesariamente los argumentos que se han planteado en términos de economía política para una potencial dolarización, pues pueden haber existido varios factores que alteraron las conclusiones del estudio, desde la selección y definición de variables hasta el hecho que ya se mencionó anteriormente: no siempre un análisis de esta clase es susceptible de validación empírica utilizando técnicas econométricas.

\section{ANÁLISIS POSKEYNESIANO INSTITUCIONALISTA}

En su trabajo Dollarization in Ecuador: A post-Keynesian institutionalist analysis, Jameson (2004) ensaya una explicación poskeynesiana institucionalista a la dolarización ecuatoriana. El autor propone una estructura teórica que contrasta como pueden confluir seis dimensiones 
de análisis - historia, instituciones, incertidumbre, causación acumulativa, poder y conflicto, y batalla de ideas- y desembocar en una decisión de política trascendental como la de escoger el régimen «adecuado» de tipo de cambio en una economía.

Uno: en cuanto a la dimensión teórica, se plantea una distinción entre el así denominado «tiempo lógico» versus el «tiempo histórico». El primero, propio de los análisis ortodoxos, asume que cualquier punto del pasado podría repetirse en el futuro y está estrechamente relacionado con el supuesto de ergodicidad. El segundo enfoque plantea que una economía está inmersa en, y es el resultado de, procesos y circunstancias históricas específicas que deben ser incluidas en cualquier análisis que se plantee al respecto de ésta.

Dos: en el apartado de las instituciones, claramente la propuesta es considerar los distintos arreglos institucionales a los que se enfrentan los agentes de una economía y cómo éstos pueden incidir en la decisión de adoptar una potencial dolarización. En este análisis se pueden incluir no solo instituciones domésticas, sino internacionales como las reglas y normas impuestos por la llamada «diplomacia del dólar del hemisferio occidental». Desde luego, considerar una dimensión institucional amplía el campo de análisis de una potencial dolarización al considerar situaciones de no equilibrio, comportamientos que no obedecen a una lógica optimizadora, cambios estructurales, entre otras.

Tres: Jameson propone analizar la inestabilidad del tipo del cambio a partir de la incertidumbre en torno a la que se forman las expectativas en los mercados financieros internacionales. Estos mercados, al no ser regulados o necesariamente equilibrados, pueden generar shocks monetario-financieros que afecten al sector real de una economía.

Cuatro: la dimensión de causación acumulativa plantea analizar un proceso de dolarización con un enfoque dinámico sistémico en el que se pueda caracterizar y analizar una economía que no necesariamente está en equilibrio. Este enfoque parte del supuesto de que los sistemas económicos son inherentemente inestables y que cualquier cambio en la estructura de una economía puede causar - o ser causado por-cambios políticos o institucionales.

Cinco: las dimensiones de poder y conflicto, y batalla de ideas, corresponden a las dos aristas a partir de las que se desarrolló la argumentación del análisis de economía política de la sección anterior, por lo que no serán replicadas en este apartado.

Seis: como se mencionó antes en el referido trabajo, Jameson (2004) aborda directamente el caso de la dolarización de la economía ecuatoriana en base a las seis dimensiones descritas arriba. Desde luego, pretender que este tipo de análisis pueda ser generalizado resultaría en sí mismo en una contradicción interna debido a los postulados sobre los que se asienta. De este modo, difícilmente se puede hablar de una validación empírica de esta propuesta de explicación al proceso de dolarización de Ecuador u otro país. Sin embargo, con seguridad es un trabajo que debe ser referenciado siempre que se pretenda entender una medida como la dolarización en un contexto más amplio que el de la ortodoxia.

\section{OTROS ASPECTOS RELEVANTES PARA LA DISCUSIÓN}

Más allá de las interminables discusiones teóricas que existen alrededor del funcionamiento de una economía monetaria - y específicamente una dolarizada-, existen varios trabajos en 
la literatura especializada que intentan explicar aspectos puntuales que resultan sumamente relevantes para entender el funcionamiento de una economía dolarizada. Desde luego, en la medida en que se entiendan los mecanismos bajo los cuales opera una economía dolarizada, se podrá plantear una discusión objetiva en cuanto a la sostenibilidad del esquema monetario. Algunos esfuerzos para entender la dolarización se han traducido en el planteamiento de modelos teóricos y otros tantos en aplicaciones empíricas para los casos más conocidos de los últimos tiempos. En esta sección se revisarán algunos de los principales aportes que se han planteado en este sentido.

\section{REVERSIBILIDAD DE LA DOLARIZACIÓN}

Cuando se habla de la potencial reversibilidad de una medida como la dolarización, es necesario distinguir entre las dos formas que han sido identificadas a lo largo del documento: privada - no oficial, de facto- - y oficial. En relación a la primera, Dean (2000) sostiene que, si bien este tipo de dolarización puede estar motivada en primera instancia por la sustitución de activos en moneda extranjera para preservar su valor, la persistencia de la dolarización puede ser explicada por una sustitución de la moneda de tal magnitud que su uso ya se haya generalizado como medio de cambio. Esta hipótesis puede ser perfectamente corroborada en los países de América Latina que han experimentado un proceso acelerado de dolarización no oficial durante períodos de inestabilidad cambiaria, en los que los actores privados optaron por moneda extranjera para proteger el valor de sus activos. Sin embargo, cuando el tipo de cambio se volvió a estabilizar y la inflación se controló, la evidencia empírica muestra que los niveles de dolarización continuaron creciendo sostenidamente.

Además, Dean basa su hipotetización acerca de la irreversibilidad de la dolarización de facto en el modelo de externalidades en red de Dowd y Greenaway (1993). En este modelo los autores proponen que el hecho de que la expansión monetaria de una economía tenga mayor o menor propensión a la sustitución por moneda extranjera depende en dos tipos de sensibilidad: del tipo de cambio a la oferta monetaria y del tipo de cambio al número de personas que utilizan la moneda extranjera. Además, un tercer factor que incidiría en esta propensión sería la medida en que la base monetaria no esté cubierta por reservas en moneda extranjera. Dean afirma que, en América Latina, las dos medidas de sensibilidad son altas y la tasa de cobertura del tercer factor es baja, por lo que estas externalidades en red producirían una irreversibilidad de la dolarización.

En cuanto a la potencial reversibilidad de una dolarización oficial, claramente es un área mucho más compleja de teorizar, en la que obligatoriamente se deberán plantear elementos de análisis propios de la economía política. Desde luego, existen unos cuantos estudios empíricos que han analizado situaciones específicas, como el caso de Argentina a inicios de los 2000, por ejemplo, pero cuyos resultados difícilmente podrían ser extrapolados a otros casos, como el de Ecuador. No obstante, varios pensadores ecuatorianos — véase, por ejemplo, los citados trabajos de Falconí y Acosta del año 2004- han planteado ya una serie de análisis relevantes y elementos para el debate, que son específicos para la realidad del país en un contexto de dolarización y potencial desdolarización. Además, es importante referirse al trabajo de Jameson (2003), quien considera que es viable reversar la adopción del dólar estadounidense en el caso 
ecuatoriano, ya que los mismos factores - sociales, institucionales, políticos - que originaron en principio el cambio de régimen monetario podrían ser los que desencadenen una potencial desdolarización. El análisis entonces, según Jameson, debería centrarse en cuáles serían los costos que se deberían asumir ante una medida de tal magnitud.

\section{EL PRESTAMISTA DE ÚLTIMA INSTANCIA}

Existe un relativo consenso en cuanto a señalar como una de las principales desventajas de la dolarización la pérdida del rol del banco central (BC) como prestamista de última instancia, al menos en el sentido tradicional. Broda y Yeyati (2003) analizan las limitaciones que enfrenta el вС en relación a su capacidad de asegurar la liquidez, tanto en países dolarizados oficialmente como en aquellos que tienen una dolarización privada. Para mitigar estos efectos, incluso en países con moneda propia, ha tomado un considerable impulso la creación de seguros de depósitos y fondos de liquidez. Sin embargo, en el citado trabajo los autores muestran como ninguno de estos mecanismos, además de otros que se proponen en la literatura relacionada, proveen una solución completa al problema que enfrenta el BC en dolarización.

En estricto sentido, la capacidad de respuesta del BC, como prestamista de última instancia bajo dolarización oficial, ante necesidades contingentes de liquidez está condicionada por el tamaño de las reservas que posee, desde luego, en dólares. No obstante, en aquellos países en los que el вС no está limitado legalmente a ser un comprador de deuda del gobierno, el BC tiene la capacidad de crear dinero ex nihilo y, consecuentemente, inyectar liquidez a la economía sin necesidad de que altere el nivel de sus reservas, al menos no en principio. Por supuesto, esta inyección de liquidez a la economía no afectará las reservas en la medida en que el dinero creado mediante compra de papeles al gobierno no sea requerido para transferencias al exterior. De ser este último el caso, las reservas se verían afectadas, con algún rezago temporal, en la proporción en que los agentes de la economía destinen el dinero creado a realizar pagos fuera del país por importaciones o pagos de deuda.

El mismo mecanismo descrito anteriormente puede ser aplicado con las entidades financieras del país sin necesidad de la referida triangulación a través del gobierno, es decir, si los arreglos institucionales lo permiten, el вС puede ser un prestamista contingente de los bancos privados creando registros electrónicos en las cuentas que estos últimos tienen en el вс. Una vez más, estas operaciones no afectarían las reservas en la medida en que estos recursos inyectados a la economía se utilicen en transacciones internas que no impliquen cambios en la preferencia por la liquidez de los agentes.

Por otro lado, en países con dolarización no oficial, la tenencia de una gran proporción de activos en dólares por parte de los bancos también reduce la capacidad del BC de fungir como prestamista de última instancia. Aunque existen alternativas para proveer liquidez y éste no sea un problema específico de los países dolarizados —oficialmente o no-, no es menos cierto que en estos últimos se requieren niveles de reservas líquidas internacionales considerablemente mayores para enfrentar crisis de liquidez de la economía (Yeyati y Sturzenegger, 2003).

Broda y Yeyati (2003) señalan la existencia de al menos dos mecanismos bajo los cuales se podría dotar de un prestamista de última instancia a un país dolarizado. Aunque quizás no sea viable en la práctica y, de serlo, podría resultar altamente costoso, sin duda la existencia de un 
acuerdo con la comunidad financiera internacional para dotar de una línea de crédito contingente al вс de un país dolarizado es una alternativa para fungir como prestamista de última instancia. Otra alternativa, en este mismo sentido, sería la de brindar la posibilidad — ¿obligatoriedad? - a los bancos privados para que puedan contratar una suerte de seguros de liquidez con bancos del exterior, liberando así la presión de esta responsabilidad al BC.

La segunda alternativa que señalan los autores es la creación de un fondo de liquidez interno que sea controlado por el mismo BC. Esta última alternativa, que ha sido implementada en países como Ecuador, por ejemplo, permite asegurar la liquidez de los bancos hasta un cierto monto, que a lo sumo sería el monto acumulado en el fondo. La implementación de un fondo de este tipo puede ser financiada por el pago de contribuciones de los bancos, por aportes desde el gobierno cuando la legislación lo permita, o con algún esquema mixto. No obstante, esta alternativa es prácticamente equivalente a tener reservas internacionales, con los consecuentes costos que esto implica.

En cualquiera de las dos alternativas anteriores, al igual que en cualquier contrato de aseguramiento, se enfrentan dos importantes problemas de riesgo moral. El primero consiste en la motivación que los bancos tendrían al estar asegurados, ante una creciente probabilidad de crisis, cubran su exposición en el mercado y contribuyan así a la crisis. En cuanto al riesgo moral del gobierno, el hecho de privatizar el rol de prestamista de última instancia podría restarle considerablemente los incentivos a monitorear y reducir el riesgo derivado de sus acciones.

\section{CRECIMIENTO, EMPLEO E INFLACIÓN}

Aunque no existe un consenso absoluto sobre las consecuencias que conlleva un proceso de dolarización en términos de crecimiento, empleo o inflación - para citar algunas de las dimensiones más relevantes-, existen importantes trabajos empíricos que dan cuenta de los impactos que una medida como la dolarización ha tenido en los países que la han adoptado. Así, por ejemplo, Soto (2009) estima un modelo econométrico con un mecanismo de corrección de errores para estimar el efecto del crecimiento del pIB, el salario real, el costo del capital y el tipo de cambio real, sobre el empleo en Ecuador posterior a la dolarización. El autor encuentra dos efectos opuestos: por un lado, existe un efecto de escala positivo derivado de un crecimiento económico sostenido en el período posterior a la adopción de la dolarización; por otro lado, se encuentra un efecto sustitución negativo: los salarios reales mínimos han aumentado mientras los precios reales de los bienes intermedios importados y el costo del capital ha disminuido, lo que implica que el trabajo se está volviendo un factor de producción más caro. Soto (2009) concluye que el nivel de empleo estará determinado por el crecimiento económico y la política del gobierno en materia de salarios mínimos.

Hallren (2014) propone una evaluación de impacto mediante un método de control sintético - modificación del método de diferencias en diferencias en el que el efecto de las características no observadas puede cambiar en el tiempo- para estimar el efecto que ha tenido la dolarización en el ingreso y la inflación. En cuanto a la segunda, al igual que ha ocurrido en otros países que han adoptado la dolarización o una medida similar de tipo de cambio fijo, en Ecuador la dolarización fue efectiva en controlar la inflación, tanto en magnitud como en volatilidad - el efecto promedio en comparación al Ecuador contrafactual «sintético» fue de 
Tabla 1. Matriz de balance modelo

\begin{tabular}{|c|c|c|c|c|c|c|c|}
\hline & HOGARES & FIRMAS & GOBIERNO & $\begin{array}{c}\text { BANCO } \\
\text { CENTRAL }\end{array}$ & $\begin{array}{c}\text { BANCOS } \\
\text { COMERCIALES }\end{array}$ & $\begin{array}{c}\text { RESTO } \\
\text { MUNDO }\end{array}$ & $\sum$ \\
\hline Inventarios & & $+I_{n}$ & & & & & $+\mathrm{I}_{\mathrm{n}}$ \\
\hline Efectivo & $+\mathrm{H}_{\mathrm{h}}$ & & & $-\mathrm{H}_{\mathrm{bc}}$ & $+\mathrm{H}_{\mathrm{b}}$ & $-\mathrm{H}_{\mathrm{rm}}$ & \\
\hline Avances de efectivo desde BC & & & & $+\mathrm{A}$ & $-\mathrm{A}$ & & \\
\hline Depósitos a la vista & $+\mathrm{M}_{1}$ & & & & $-\mathrm{M}_{1}$ & & \\
\hline Depósitos a plazo & $+\mathrm{M}_{2}$ & & & & $-\mathrm{M}_{2}$ & & \\
\hline Deuda de corto plazo & $+\mathrm{DC}_{\mathrm{h}}$ & & $-\mathrm{DC}$ & $+\mathrm{DC}_{\mathrm{bc}}$ & $+\mathrm{DC}_{\mathrm{b}}$ & $+\mathrm{DC}_{\mathrm{rm}}$ & \\
\hline Deuda de largo plazo & $+\mathrm{DL}_{\mathrm{h}}$ & & $-\mathrm{DL}$ & $+\mathrm{DL}_{\mathrm{bc}}$ & & $+\mathrm{DL}_{\mathrm{rm}}$ & \\
\hline Créditos & & $-\mathrm{L}$ & & & $+\mathrm{L}$ & & \\
\hline Reservas internacionales & & & & $+\mathrm{R}$ & & $-\mathrm{R}$ & \\
\hline Depósitos en el BC & & & $+\mathrm{DBC}_{\mathrm{g}}$ & $-\mathrm{DBC}$ & $+\mathrm{DBC}_{\mathrm{b}}$ & & \\
\hline Balance & $-\mathrm{V}$ & & $+\mathrm{DN}$ & & & & $-I_{n}$ \\
\hline$\sum$ & 0 & 0 & 0 & 0 & 0 & 0 & 0 \\
\hline
\end{tabular}

-1,04 puntos en escala logarítmica para el período 2006-2010-. En contraposición a otros estudios similares, Hallren (2014) encuentra que la dolarización en el caso ecuatoriano no ha tenido efecto en el ingreso real per cápita. Finalmente, el autor concluye que se requieren ajustes estructurales que flexibilicen el mercado laboral e impongan disciplina fiscal para que la dolarización sea sostenible en el largo plazo.

En relación al crecimiento del PIB, Edwards y Magendzo (2003) un modelo de efectos de tratamiento para contrastar el desempeño de países dolarizados con el de aquellos que tienen moneda propia. Los autores encuentran que el crecimiento económico es ligeramente menor, aunque no estadísticamente significativo, en los países dolarizados que en los países con moneda propia. Además, en los países que han adoptado esta medida, el estudio encuentra que la volatilidad de la tasa de crecimiento del PIB es considerablemente mayor que en los países con moneda propia.

\section{PREFERENCIA EXTERNA DE LIQUIDEZ DOMÉSTICA}

Más allá de la obvia diferencia que existe entre un régimen dolarizado y uno con tipo de cambio fijo en cuanto a la disponibilidad de moneda propia, existen otros dos aspectos diferenciadores que resultan medulares para esta discusión. El primero de ellos ya se mencionó en la sección anterior: la existencia de un prestamista de última instancia. En una economía que ha optado por el tipo de cambio fijo, si bien la capacidad de emisión está limitada por las reservas internacionales, las transacciones domésticas no están sujetas a esta restricción, de manera que el вС puede responder ante un incremento en la demanda de dinero por parte de los hogares, firmas o bancos. En una economía con tipo de cambio fijo el BC incluso puede mitigar las restricciones fiscales propias de un régimen con tipo de cambio fijo, pudiendo actuar también como prestamista de última instancia del gobierno. Por otro lado, en una economía dolarizada el déficit fiscal ya no está financiado de forma automática, pues la capacidad del BC de operar como prestamista del gobierno está limitada por su hoja de balance. 
La segunda diferencia de fondo, que ha sido tratada de forma muy escueta en la literatura relacionada, se refiere a la preferencia externa de liquidez doméstica. En un esquema de tipo de cambio fijo, al existir físicamente una moneda local, su utilización - y demanda- está limitada al ámbito doméstico. En este caso, la moneda local no es necesariamente apetecible para el sector externo, por lo que solamente los cambios en la preferencia doméstica de liquidez podrían afectar a la liquidez global de la economía. En contraparte, cuando un país sustituye la moneda nacional por una moneda extranjera - que, desde luego, deberá ser una moneda fuerte para que la medida tenga sentido- existe un sector externo que tiene «interés» en la moneda que ha adoptado el país en cuestión. Más aún, si la moneda que se adopta es el dólar, que es una moneda apetecida en prácticamente cualquier lugar del mundo, la demanda externa de dólares - preferencia externa de liquidez doméstica - puede afectar la liquidez global de la economía, sobre todo por parte de países cuyas monedas estén en períodos de depreciación respecto del dólar.

\section{ENDOGENEIDAD LIMITADA}

Como ya se mencionó en la cuarta sección, uno de los aspectos que más debate ha generado entre las distintas escuelas del pensamiento es la neutralidad o no neutralidad del dinero. Estas discusiones, desde luego, han derivado en las distintas posturas existentes respecto de la endogeneidad o exogeneidad del dinero. Este debate se ha centrado en un tipo específico de endogeneidad: aquel que hace referencia a la creación de dinero - oferta monetaria-. Sin embargo, como afirma Palley (2002), existen al menos otras diez clases de endogeneidad que se pueden identificar en la literatura de las distintas escuelas del pensamiento: evolutiva - mengeriana-, cuantitativa neoclásica, fusión entre evolutiva y cuantitativa neoclásica, del comportamiento del BC, fiscal, de selección de portafolio y multiplicador monetario, poskeynesiana - de crédito-, del intermediario financiero desde el lado de la oferta, de la distribución sectorial del dinero, de economía abierta.

En una economía con moneda propia, sea cual fuere el nivel de flexibilidad o rigidez de su tipo de cambio, se pueden identificar de forma relativamente objetiva varios - o quizás todas - tipos de endogeneidad de los anteriormente mencionados. No obstante, en una economía dolarizada esta identificación no resulta trivial. El comportamiento del BC y del sector fiscal, por ejemplo, puede tener una dinámica endógena en la medida en que las condiciones domésticas, como la preferencia por la liquidez o las necesidades de financiamiento, no cambien. Sin embargo, esta dinámica endógena tiene un techo, que está determinado por la cantidad de reservas internacionales que tenga el país. En esta misma discusión se puede agregar la endogeneidad propia de una economía abierta o la endogeneidad poskeynesiana. Esta última, que quizás es la que más se ha posicionado partiendo de la escuela circuitista, es cada vez menos resistida cuando se analizan economías con moneda propia. En una economía dolarizada la dinámica endógena del crédito no resulta del todo evidente como en una economía con moneda propia, pues la capacidad de crear dinero ex nihilo por parte de los bancos - vía crédito- está limitada por los requerimientos de reservas mínimas del BC, las cuales a su vez terminan dependiendo una vez más en las reservas internacionales. 
Tabla 2. Matriz de transacciones de modelo

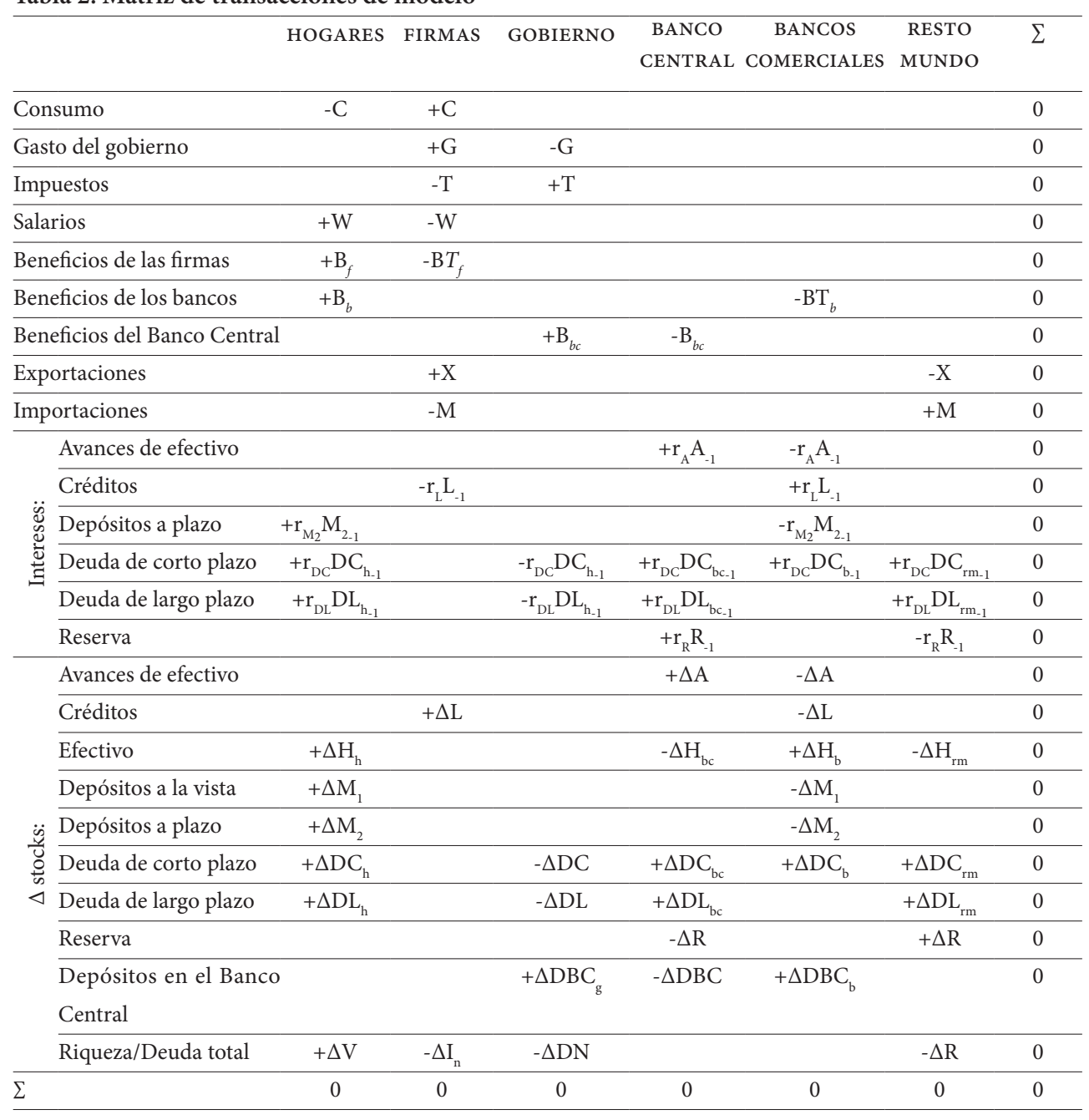

Fuente: autor.

\section{EL SISTEMA FINANCIERO}

El comportamiento del sistema financiero bajo un régimen dolarizado es otro de los aspectos sumamente relevantes para entender este esquema monetario y un eventual abandono del mismo. Gale y Vives (2001) analizan los costos y beneficios de la dolarización, en tanto que herramienta para proveer «disciplina de mercado» para la banca. Los autores plantean una modificación de los modelos de Diamond y Dybvig, originalmente planteados en los ochenta para corridas bancarias. En estos modelos se supone la existencia de un continuo de agentes optimizadores de su utilidad, una proporción de los cuales es impaciente en cuanto 
a sus preferencias de liquidez. Uno de los principales aportes de los autores es la incorporación al modelo del riesgo moral al estilo Krugman. Entre los principales hallazgos del estudio se encuentra una categorización de países en los cuales la dolarización sería beneficiosa en términos de mayor estabilidad el sistema bancario, entre los cuales se encuentra precisamente Ecuador. Además, dadas las condiciones del país antes de sustituir la moneda local, Ecuador estaría entre los países cuya adopción de la dolarización implicaría los menores costos, de entre los analizados.

Quispe-Agnoli y Whisler (2006), por otro lado, plantean una reseña empírica de algunos indicadores de desempeño de la banca, antes y después de la dolarización en Ecuador y El Salvador. Los autores utilizan datos de panel para los bancos de ambos países, entre 1995 y 2004, para evaluar si la dolarización tuvo un efecto en el desempeño de la banca. El estudio concluye que, aunque los dos países adoptaron la dolarización por distintas razones, ambos han experimentado considerables mejoras en cuanto a regulación de la banca y la estabilidad del sistema financiero. Según los autores, la dolarización en los dos países jugó un papel determinante en cuanto a mejorar la liquidez del sistema financiero y la calidad de los activos.

Arellano y Heathcote (2010) proponen un modelo teórico de optimización intertemporal para evaluar si la dolarización brinda mejores condiciones de acceso a los mercados de financiamiento internacional para los países que adopten esta medida. En su modelo los agentes deciden entre consumir o tener efectivo - la única forma de ahorro considerada en el modelo-. Además, existe una firma representativa que produce una cantidad estocástica de bienes y un gobierno limitado que decide sus políticas de tal suerte que maximiza el bienestar de los consumidores. Uno de los principales hallazgos del estudio es que los efectos de la dolarización en la integración de un país con los mercados financieros internacionales, así como en sus indicadores de bienestar, depende fuertemente en el nivel de las tasas de interés externas. Así, un mercado financiero internacional con bajas tasas de interés hace especialmente atractiva a la dolarización, pues se convierte en un instrumento de bajo costo para mitigar las fluctuaciones. De este modo, la pérdida de la emisión monetaria como instrumento de política, según los autores, se ve en contrapeso con la mejor capacidad de adquirir endeudamiento de bajo costo.

\section{LAS RESERVAS INTERNACIONALES}

Las características de una economía dolarizada referidas en los apartados anteriores han puesto de manifiesto las posibles fuentes de vulnerabilidad de estos sistemas. Estos aspectos, aunque son las causas o los efectos directos del estado de salud del sistema monetario-financiero, generalmente no son sintomáticos en sí mismos. La cara visible del sistema en una economía de este tipo la constituyen las reservas internacionales — la punta del iceberg-. Ante la ausencia de mecanismos e instituciones tradicionales que permitan ejercer de forma activa la política monetaria en un país sin moneda propia, las reservas son las encargadas de soportar la presión que generan los demás sectores de la economía. Los cambios en la preferencia por la liquidez doméstica, sea interna o externa, así como las operaciones de financiamiento del déficit fiscal a través de transacciones locales, se ven reflejados en última instancia en variaciones de la reserva internacional. 
Figura 1. Resultados del escenario 1

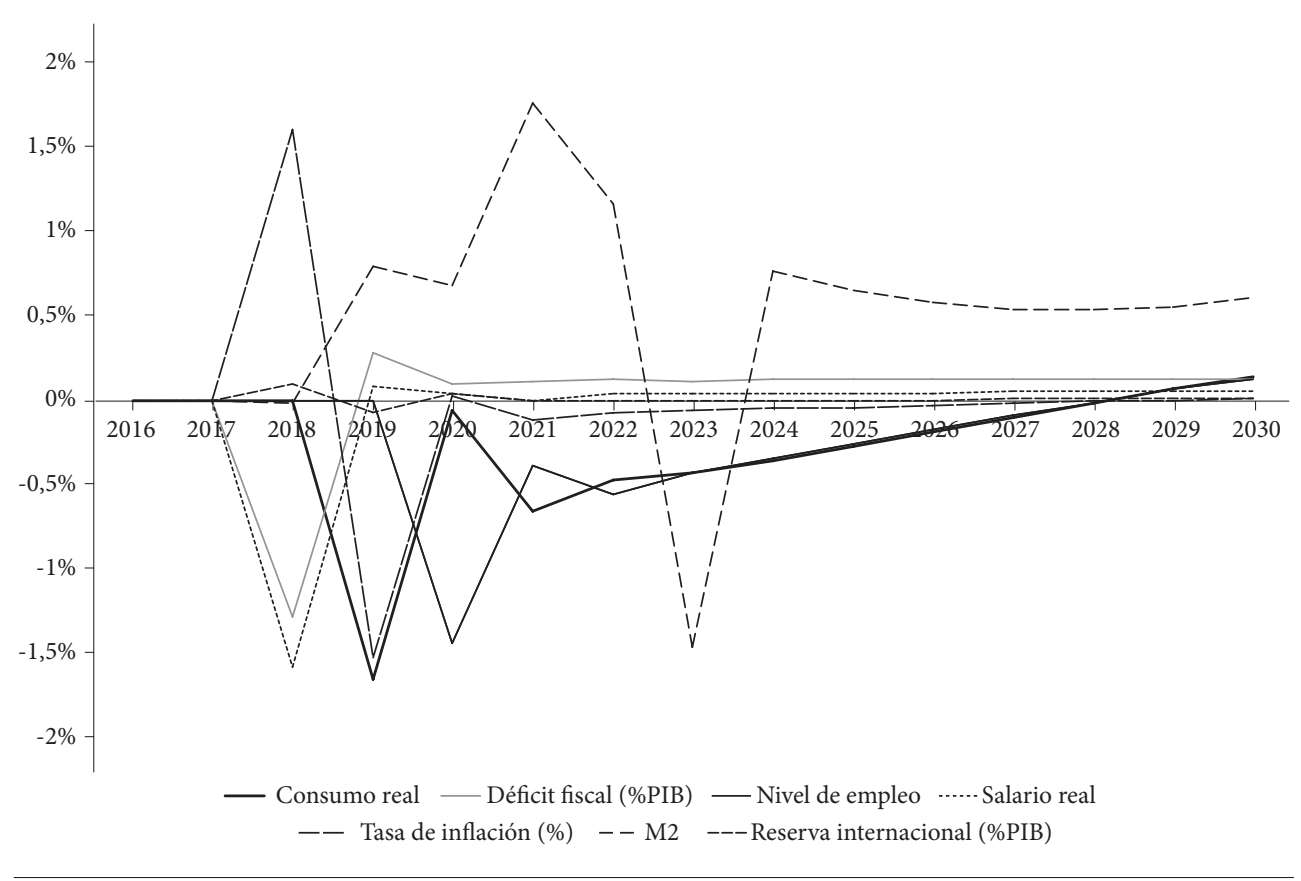

Fuente: modelo.

\section{UN MODELO STOCK-FLOW CONSISTENT PARA UNA ECONOMÍA DOLARIZADA}

Los modelos stock-flow consistent ( $\mathrm{SFC}$ ) son herramientas de modelación macroeconómica que se construyen en base a tres elementos (Dos Santos, 2002): i) una matriz de stocks en la que se encuentran los balances - activos y pasivos - de los sectores institucionales considerados en la modelación, ii) una matriz de flujos en la que se determinan los orígenes y destinos de todas las transacciones que se efectúan en una economía, de manera que no existan «agujeros negros» (Godley, 1996), es decir, cada flujo viene de algún lado y va a algún lado, y iii) un conjunto de ecuaciones que determinan las reglas de actualización de los stocks y la dinámica de las variables consideradas. Estos modelos tienen la potencialidad de explicar de forma clara la interacción entre la parte real y la financiera de la economía que está siendo modelada, permitiendo así «rastrear» con precisión las relaciones entre los distintos sectores.

En esta sección se presenta la estimación de un modelo SFC para una economía pequeña, abierta y dolarizada. El desarrollo se hizo tomando como base - y ampliándolo con las particularidades de una economía como la mencionada - el modelo insout de Godley y Lavoie (2007) (ver Tablas 1 y 2).

\section{CARACTERÍSTICAS DE LOS SECTORES INSTITUCIONALES}

En lo que sigue se explicarán las principales características del modelo, en términos de los sectores institucionales considerados. 
Gobierno: la variable de decisión residual del gobierno es la emisión de deuda de corto plazo, con esto financia el déficit fiscal; provee deuda de largo plazo por demanda de los hogares; ingresos por mediante impuestos sobre las ventas de las firmas - que los recogen indirectamente de los hogares-; dirige una proporción no constante de su gasto a comprar la producción de las firmas.

Banco Central: eventualmente puede tener beneficios ya que sus activos generan retornos, pero sus pasivos no pagan interés, los beneficios se distribuyen al gobierno; la variable de decisión residual del вС corresponde a los avances de efectivo que entrega a los bancos privados - no puede emitir moneda-; es el comprador residual de la deuda emitida por el gobierno que no ha sido adquirida por los hogares o los bancos comerciales; el BC tiene entre sus pasivos una cuenta de depósitos del gobierno y las firmas, ésta es la cuenta que se utiliza para cuadrar la hoja de balance del вс cuando se realizan avances de efectivo.

Firmas: las firmas producen en función de sus ventas esperadas más la diferencia entre los inventarios esperados y los inventarios realizados del período anterior, más un factor proporcional al crédito demandado no correspondido del período anterior; sus decisiones de producción pasan por la cantidad de trabajo a contratar y deciden las importaciones requeridas para la producción; fijan los precios en función de un mark-up sobre los costos unitarios históricos - ecuación kaleckiana de formación de precios-; los beneficios de las firmas se distribuyen a los hogares; recogen impuestos indirectamente de los hogares y pagan impuestos directamente al gobierno; la variable residual de decisión son los créditos que obtienen de los bancos - necesidades de financiamiento-; cuando las firmas son sujetas de racionamiento de crédito, sus inventarios no pueden «realizarse» en forma de beneficios, en su totalidad, existiendo así dos tipos de inventarios: los que son correspondidos por la oferta crediticia y los inventarios físicos no realizados; responden de forma irrestricta a la demanda exógena de exportaciones.

Hogares: su portafolio de decisión tiene 5 activos: efectivo, depósitos a la vista, depósitos a plazo, deuda pública de corto plazo y deuda pública de largo plazo; sus decisiones de inversión las hacen en función de las tasas de interés nominales de los activos; reciben ingresos de los retornos de sus inversiones; la variable residual de decisión de los hogares es el monto de depósitos a la vista.

Bancos comerciales: ocasionalmente obtienen avances en efectivo del bc; además de los créditos, tienen otros tres activos: reservas en efectivo en el вс, depósitos en el вс y deuda de corto plazo del gobierno - bills - ; deciden las tasas que pagan en los depósitos a plazo y las que cobran en los créditos, dentro de un límite máximo establecido por el вC; las variables de decisión residuales son la tenencia de deuda pública y los avances de efectivo que obtienen del BC; tienen un rol más trascendental que el de únicamente decidir las asignaciones de su portafolio de inversiones; no necesariamente responden a las necesidades de financiamiento de las firmas; los bancos deciden sobre las tasas de interés de créditos y depósitos - price-takers de las tasas de avances del вС y deuda de corto y largo plazo, y price-makers de las tasas de créditos y depósitos- procurando un margen de beneficios esperado y también en respuesta a un ratio de liquidez; los bancos pueden racionar el crédito si el techo máximo de las tasas de interés fijado por el вС no permite satisfacer sus expectativas de rentabilidad.

Resto del mundo: es el comprador residual de la deuda de largo plazo del gobierno; paga una tasa de interés fija sobre el stock de reserva internacional; las variaciones de la reserva 
Figura 2. Resultados del escenario 2

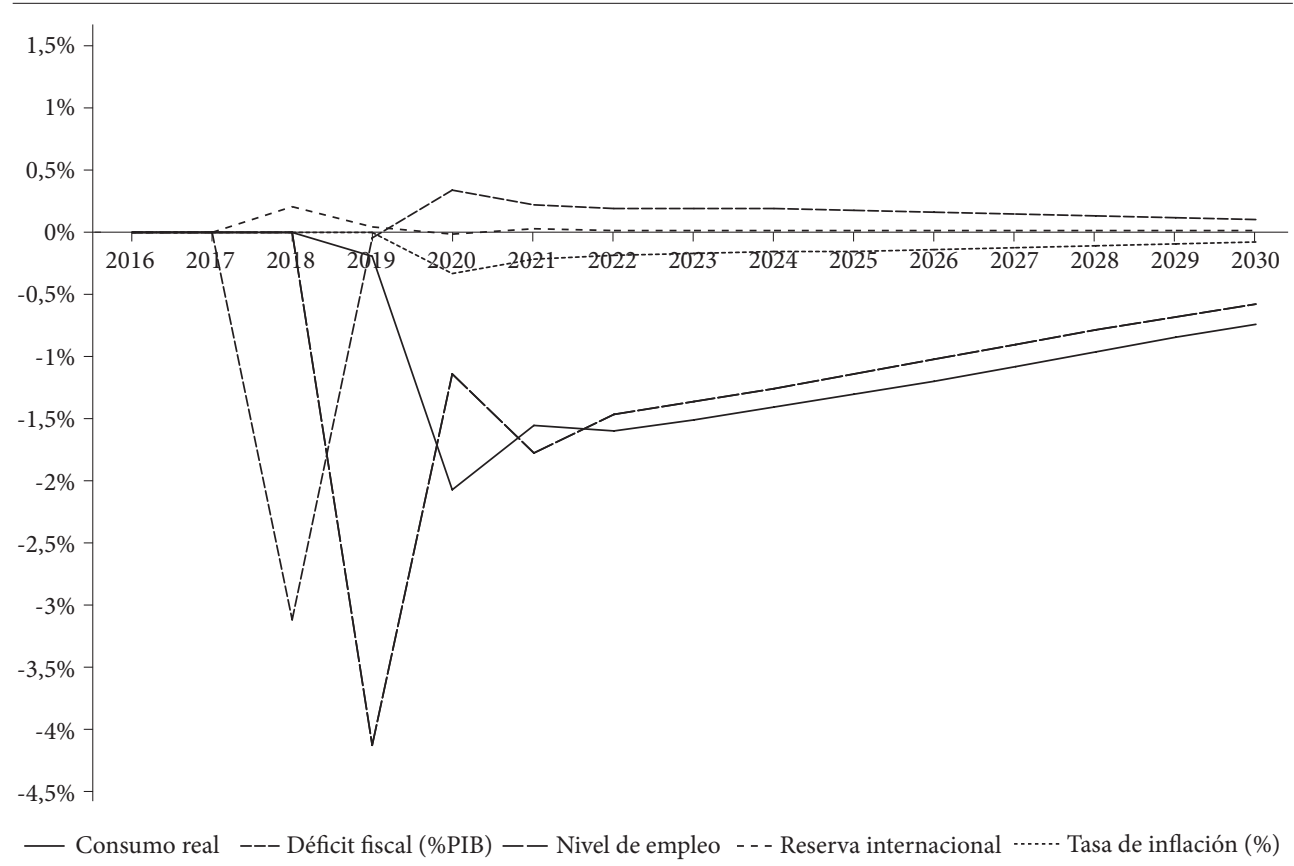

Fuente: modelo.

internacional están determinadas, además de factores internos como la preferencia por la liquidez, por factores proporcionales de la balanza comercial con el resto del mundo y del saldo de deuda pública; el sector externo fija una demanda exógena de exportaciones nacionales; satisface la demanda de importaciones de las firmas locales sin restricción; al ser una economía dolarizada, tiene como un pasivo la mayor parte del dinero circulante, pues solamente la moneda fraccionaria que emite el вС es parte del pasivo de este último.

\section{SIMULACIONES}

El modelo presentado en este trabajo es netamente teórico. No obstante, debido a la cantidad y complejidad de las ecuaciones involucradas, es muy difícil realizar ejercicios de estática o dinámica comparativa para evaluar medidas de política. Debido a esto se utilizarán simulaciones para entender los efectos de un grupo de medidas de política. En primer lugar, es necesario definir el estado estacionario que servirá de base para correr las simulaciones. El supuesto de dicho estado estacionario en este modelo es que el valor real de las ventas $s^{\star}$ es igual al output real $y^{\star}$.

$$
y^{*}=s^{*}(5)
$$

Por otro lado, del comportamiento explicado de los sectores institucionales, se concluye que los impuestos a precios corrientes corresponden a una fracción de las ventas a precios corrientes: 


$$
T=p s \frac{\tau}{1+\tau^{\prime}}
$$

donde $\tau$ es el tipo impositivo de la economía. Despejando, el valor real de las ventas es igual a:

$$
s=\frac{T(1+\tau)}{p \tau}
$$

Además, de la ecuación de consistencia contable del gobierno, se puede ver que:

$\mathrm{T}+\mathrm{BbC}+\Delta \mathrm{DBCg}=\mathrm{G}+\mathrm{rDCDC}-1+\mathrm{rDLDL}-1+\Delta \mathrm{DC}+\Delta \mathrm{DL}$

Desde luego, en el estado estacionario, $\Delta \mathrm{DBCg}=\Delta \mathrm{DC}=\Delta \mathrm{DL}=0$. Sin necesidad de mayor argumentación, se puede asumir que los beneficios distribuidos al gobierno por parte del banco central $\mathrm{B}_{\mathrm{bc}}$ también son nulos en el estado estacionario. De esto se concluye que:

$\mathrm{T}=\mathrm{G}+\mathrm{r}_{\mathrm{DC}} \mathrm{DC}^{*}+\mathrm{r}_{\mathrm{DL}} \mathrm{DL}^{\star}(9)$

Finalmente, la ecuación de equilibrio que sirve de base para las simulaciones sería:

$$
y^{*}=\left(G+r_{D C} D C^{*}+r_{D L} D L^{*}\right) \frac{1+\tau}{p \tau} .
$$

\section{ESCENARIOS}

Con base en la especificación descrita del modelo, se han simulado dos escenarios. Escenario 1: se incrementa la tasa impositiva general en dos puntos porcentuales y se regresa al valor de la línea base después de un año. Escenario 2: se reduce el consumo local del gobierno - compras a firmas- en un $20 \%$.

A continuación se presentarán los resultados en algunas variables de interés, posteriores a la implementación del choque correspondiente a cada escenario simulado. En todos los casos, el año inicial considerado para la simulación es 2016 y se asume que los choques se producen en el año 2018. Se muestran los resultados de corto y largo plazo de las variables más relevantes en una ventana de tiempo que se extiende hasta el año 2030. Es necesario recalcar que no se trata de un modelo de proyecciones; estas carecerían de objetividad con un horizonte temporal así. Éste es un modelo explicativo que permite entender las respuestas de las principales variables que relacionan los sectores real y financiero-monetario, ante determinados shocks en ciertos parámetros de interés.

La figura 1 muestra los resultados en algunas variables macroeconómicas relevantes posteriores a un incremento en la tasa impositiva general -impuestos- equivalente a dos puntos porcentuales. En este escenario se supone que la medida es reversada después de un año de su implementación. Estos valores no deben interpretarse en términos absolutos, sino como el porcentaje de «desviación» respecto a la tendencia que habrían tenido en la línea base, en caso de que no hubiese existido el choque.

Finalmente, la figura 2 muestra los resultados, siempre interpretados como el porcentaje de cambio respecto a la línea base, del segundo escenario. 


\section{CONCLUSIONES}

Los resultados del escenario de incremento de la tasa impositiva general en dos puntos porcentuales, y su reducción en un año, muestran que la tendencia de corto plazo del producto real —output - cae un año, pero se recupera al estado inicial rápidamente y se mantiene en el largo plazo. De igual forma se presenta una recuperación más rápida del salario real y nivel de empleo. En cuanto a la liquidez global de la economía, esta mejora y solo tiene un efecto rebote negativo en el mediano plazo, sin embargo, vuelve a recuperarse en el largo plazo.

En el segundo escenario se evidencia que, si bien una contracción en gasto del gobierno puede reducir el déficit en aproximadamente $3 \%$ durante un par de años, este efecto prácticamente desaparece al tercer año. Sin embargo, los efectos de una medida como ésta, tanto en el output como en el empleo y el consumo, no solamente son mayores en magnitud, sino que sus efectos son duraderos en el mediano plazo - el tiempo de regreso a los niveles de la línea base es de aproximadamente 15 años-. Es decir, una medida de austeridad de este tipo afectaría la demanda agregada en mediano plazo, con efectos considerables en el producto real.

La discusión respecto de la sostenibilidad de la dolarización es un debate abierto. Existen varios elementos teóricos, que responden a diversas escuelas del pensamiento económico, que deben ser tomados en cuenta para realizar un análisis objetivo en relación a este tema. Los escasos ejemplos de economías que han optado por esta vía hacen que las evaluaciones empíricas del funcionamiento de este esquema monetario sean bastante limitadas. En consecuencia, el ejercicio de determinar las potenciales vulnerabilidades de la dolarización dependerá en gran medida de la realidad propia del país que se analice. Es necesario, por tanto, desarrollar herramientas específicas para cada realidad que se pretenda entender y modelar.

Los modelos SFC brindan una gran flexibilidad, dentro de un riguroso esquema de consistencia contable, para reflejar los distintos comportamientos de los agentes/sectores institucionales que se pretendan incorporar. En este documento se han presentado algunos resultados preliminares obtenidos de la estimación de un modelo SFC teórico para una economía dolarizada y abierta. Sin embargo, es necesario aún un ejercicio de modelación mucho más detallado y aplicado que permita entender las limitaciones específicas del esquema monetario de un país dolarizado.

\section{REFERENCIAS}

Acosta, A. (2004). Dolarización o desdolarización, ¡esa no es toda la cuestión! Íconos, 19, 54-65.

Acosta, A. (2012). Breve historia económica del Ecuador. Quito, Ecuador: Corporación Editora Nacional.

Arellano, C. and Heathcote, J. (2010). Dollarization and financial integration. Journal of Economic Theory, 145(3), 944-973.

Bell, S. (2003). Common currency lessons from Europe. En L.-P. Rochon y M. Seccareccia (Eds.), Dollarization: Lessons from Europe and the Americas. London, uk: Routledge.

Broda, C. and Yeyati, E. L. (2003, March 19). Dollarization and the lender of last resort. Recuperado de https://pdfs. semanticscholar.org/8c7b/b6759e85d93172b35d444ffd4265756ef94a.pdf

Caprio, G., Honohan, P., and Vittas, D. (2002). Financial sector policy for developing countries: a reader. (s. d.): World Bank Publications. 
Dean, J. W. (200o). De facto dollarization. En Conference To Dollarize or not to Dollarize: Exchange Rate Choices for the Western Hemisphere, Ottawa, Canada, 4, (s. d).

Dos Santos, C. H. (2002). Notes on the stock-flow consistent approach to macroeconomic modeling. Three Essays in Stock-Flow Consistent Macroeconomic Modeling. (s. d.).

Dowd, K. and Greenaway, D. (1993). Currency competition, network externalities and switching costs: Towards an alternative view of optimum currency areas. The Economic Journal, 103(420), 1180-1189.

Edwards, S. and Magendzo, I. (October, 2003). Strict dollarization and economic performance: An empirical investigation. Technical report. National Bureau of Economic Research, 8, 351-363.

Falconí, F. (2004). Dolarización y desdolarización: elementos para el debate. íconos, 19, 22-24.

Frieden, J. A. (2003). The political economy of dollarization: domestic and international factors. (s. d.).

Gale, D. M. and Vives, X. (2001). Dollarization, bailouts and the stability of the banking system. (s. d.).

Godley, W. (1996). Money, income and distribution: an integrated approach. Levy Economics Institute, Working Paper, 167, (s. d.).

Godley, W. and Lavoie, M. (2007). Monetary economics. Hampshire, uk: Palgrave Macmillan Basingstoke.

Hallren, R. J. (2014). The impact of dollarization and currency boards on income and inflation: A synthetic control analysis. Available at SSRN 2486253.

Humphrey, T. M. (1974). The quantity theory of money: its historical evolution and role in policy debates. FRB Richmond Economic Review, 6o, 2-19.

Jameson, K. P. (2003). Is it possible to de-dollarize? The case of Ecuador. International Journal of Political Economy, 33(1), 42-60.

Jameson, K. P. (2004). Dollarization in Ecuador: A post-Keynesian analysis. (Technical report). University of Utah, UsA.

Kenen, P. (1969). The theory of optimum currency areas: an eclectic view. Monetary problems of the international economy, (s. d.), 41-6o.

Levy, E. and Sturzenegger, F. (2003). Dollarization: Debates and Policy Alternatives. Massachusetts, usa: Massachusetts Institute of Technology.

Marx, K. (s. f.). Capital, (s. d.).

Mundell, R. A. (1961). A theory of optimum currency areas. The American Economic Review, 51(4), 657-665.

Palley, T. I. (2002). Endogenous money: what it is and why it matters. Metroeconomica, 53(2), 152-180.

Quispe-Agnoli, M. and Whisler, E. (2006). Official dollarization and the banking system in Ecuador and El Salvador. Economic Review-Federal Reserve Bank of Atlanta, 91(3), 55.

Rose, A. K. (2000). One money, one market: the effect of common currencies on trade. Economic policy, 15(30), 08-45.

Sachs, J. y Larrain, F. (2000). ¿Por qué la dolarización es más una camisa de fuerza que una salvación? En A. Acosta y J. c. Juncosa (Eds.), Dolarización: informe urgente. Quito, Ecuador: Ildis y Abya-Yala.

Say, J. B. (1836). A treatise on political economy: or the production, distribution, and consumption of wealth. Philadelphia, UsA: Grigg \& Elliot.

Soto, R. (2009). Dollarization, economic growth, and employment. Economics letters, 105(1), 42-45.

Yeyati, E. L. and Sturzenegger, F. (2003). Dollarization: A primer. (s. d.). 